A

RESEARCH

\title{
Autoimmune thyroid disease correlates to islet autoimmunity on zinc transporter 8 autoantibody
}

\author{
Yun Cai ${ }^{1} *$, Jieni Yan ${ }^{1} *$, Yong Gu ${ }^{1}$, Heng Chen ${ }^{1}$, Yang Chen ${ }^{1}$, Xinyu Xu ${ }^{1}$, Mei Zhang ${ }^{1}$, Liping $\mathrm{Yu}^{2}$, Xuqin Zheng ${ }^{1}$ and \\ Tao Yang ${ }^{1}$ \\ 1'Department of Endocrinology, The First Affiliated Hospital of Nanjing Medical University, Nanjing, China \\ ${ }^{2}$ Barbara Davis Center for Diabetes, University of Colorado School of Medicine, Aurora, Colorado, USA \\ Correspondence should be addressed to X Zheng or T Yang: zhengxuqin@njmu.edu.cn or yangt@njmu.edu.cn \\ *(Y Cai and J Yan contributed equally to this work)
}

\begin{abstract}
Objective: The most common coexisting organ-specific autoimmune disease in patients with type 1 diabetes mellitus (T1DM) is autoimmune thyroid disease (AITD). However, there have been few clinical reports based on a large population about the prevalence of zinc transporter 8 autoantibody (ZnT8A) and other islet autoantibodies in AITD patients. We aimed to explore the presence of islet autoantibodies, ZnT8A, glutamic acid decarboxylase autoantibodies (GADA) and insulinoma-associated antigen 2 autoantibodies (IA-2A) compared with thyroid autoantibodies, thyroid peroxidase autoantibodies (TPOAb) and thyroglobulin autoantibodies (TGAb) and thyrotropin receptor autoantibodies (TRAb) in patients with Graves' disease (GD), Hashimoto's thyroiditis (HT) and T1DM patients with AITD.

Methods: Totally, 389 patients with GD, 334 patients with HT, 108 T1DM patients with AITD and 115 healthy controls $(\mathrm{HC})$ were recruited in the study. Islet autoantibodies (ZnT8A, GADA and IA-2A) were detected by radioligand binding assay. Thyroid autoantibodies, TPOAb and TGAb were detected by chemiluminescence assay, and TRAb was detected by RIA.

Results: The prevalence of ZnT8A, GADA and IA-2A was higher in GD and HT patients than that of HC (ZnT8A: GD 8.48\%, HT 10.8\% vs HC 1.74\%; GADA: GD 7.46\%, HT 7.74\% vs HC $0.870 \%$; IA-2A: GD 4.88\%, HT 3.59\% vs HC 0\%; All $P<0.05$ ) but lower than that of T1DM subjects with AITD (ZnT8A: 42.6\%; IA-2A: 44.4\%; GADA: 74.1\%; all $P<0.0001$ ). Conclusions: An increased prevalence of ZnT8A as well as GADA and IA-2A was found in both GD and HT patients, indicating that there is a potential link between thyroid autoimmunity and islet autoimmunity.
\end{abstract}

\section{Key Words}

- autoimmune thyroid disease

- type 1 diabetes mellitus

- zinc transporter 8 autoantibody

- thyroid peroxidase autoantibodies

\section{Introduction}

Autoimmune thyroid disease (AITD) is a common organ-specific autoimmune disorder which includes two main clinical diseases: Graves' disease (GD) and Hashimoto's thyroiditis (HT). Both GD and HT share the immunologic manifestation of the presence of circulating autoantibodies such as thyrotropin receptor autoantibodies (TRAb), thyroid peroxidase autoantibodies (TPOAb) and thyroglobulin autoantibodies (TGAb). TRAb occurs predominantly in GD and plays a key role in Graves' hyperthyroidism, while TGAb and TPOAb are more characteristic for HT, which is correlated with thyroidal damage and lymphocytic inflammation $(1,2)$.

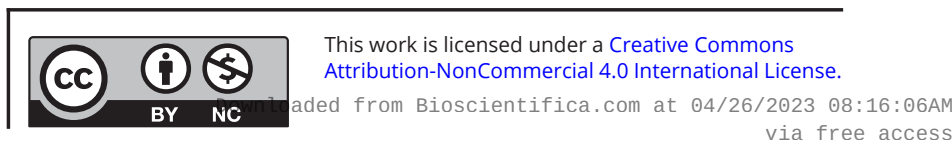


Individuals with AITD may be associated with other autoimmune diseases, of which type 1 diabetes mellitus (T1DM) is the most frequent $(3,4,5)$. The phenotype is referred as autoimmune polyendocrine syndrome type II (APS II) when T1DM and AITD occur in the same individual, even sometimes coexist in the same family (6, 7). T1DM, as well as AITD, develops through a complex interaction of genetic and environmental risk factors which result in autoimmune destruction of the specific organs involving humoral and cellular immune responses $(1,2,4,8,9)$. Many studies have showed the prevalence rate of thyroid autoantibodies ranging from $3.7-35 \%$ in T1DM patients, some of which suggested the associations between thyroid autoantibodies and islet autoantibodies $(10,11,12,13,14)$. However, little work has been done on the anti-islet autoimmune status in non-diabetic patients with $\operatorname{AITD}(15,16,17,18,19,20,21,22,23)$. As summarized in Supplementary Table 1 (see section on supplementary materials given at the end of this article), the prevalence rates of glutamic acid decarboxylase autoantibodies (GADA), islet cell autoantibodies (ICA), insulinoma-associated antigen 2 autoantibodies (IA-2A) and insulin autoantibodies (IAA) in patients with AITD were either slightly or significantly higher than those in healthy controls (HC), indicating the distinct value of studying islet immune status in AITD.

Zinc transporter 8 autoantibody (ZnT8A) is the recently identified new marker of autoimmunity in diabetes (24). Furthermore, previous studies have demonstrated that the presence of ZnT8A predisposes non-obese adult-onset diabetes patients to AITD (25). However, there has been no clinical study based on a large population focusing on ZnT8A in both GD and HT patients without diabetes mellitus (DM).

Our study aims to measure three islet autoantibodies, ZnT8A, GADA and IA-2A in Chinese Han patients with GD and HT (but without DM) as well as to investigate the relationship between islet autoantibodies and thyroid autoantibodies. In addition, the differences in prevalence among AITD, T1DM patients with AITD (GD or HT) and HC were compared to explore the anti-islet autoimmune status. The data gained in this analysis are supposed to provide a rationale for screening programs for active casefinding strategies in specialized centers.

\section{Materials and methods}

\section{Subjects}

There were 389 Chinese patients with GD (284 female and 105 male; aged $38.9 \pm 14.0$ years; BMI $22.1 \pm 3.2 \mathrm{~kg} / \mathrm{m}^{2}$ ) and 334 patients with HT (301 female and 33 male; aged $41.6 \pm 14.3$ years; BMI $22.7 \pm 3.4 \mathrm{~kg} / \mathrm{m}^{2}$ ), who have not been diagnosed with DM before, recruited in our study. These two autoimmune thyroid diseases were diagnosed clinically by endocrinologists and confirmed by clinical symptoms and abnormal levels of thyroid hormones and autoantibodies to TRAb, TPOAb and/or TGA as well as ultrasound examination. One hundred and eight patients with T1DM and AITD (67 female and 41 male; aged $32.7 \pm 17.5$ years; BMI $20.8 \pm 3.4 \mathrm{~kg} / \mathrm{m}^{2}$ ) were recruited in our study. Individuals with T1DM were diagnosed according to the diagnosis criteria of the World Health Organization and American Diabetes Association. One hundred and fifteen healthy controls ( 87 female and 28 male; aged $44.7 \pm 12.8$ years; BMI $21.4 \pm 2.6 \mathrm{~kg} / \mathrm{m}^{2}$ ) were also included as HC groups. Sera of patients with GD or HT and $\mathrm{HC}$ were obtained in the endocrinology department of the First Affiliated Hospital of Nanjing Medical University from October 2010 to September 2013, and sera of T1DM with AITD patients were obtained at 26 centers in China from June 2010 to Oct 2012. The clinical characteristics of all subjects are summarized in Table 1 . Blood samples were collected for thyroid function, thyroid autoantibodies detection and further islet autoantibodies assay. However, blood samples for HLA genotyping and fasting C-peptide were not collected in solely AITD patients.

Our study was approved by the appropriate ethical committees, and informed consent was obtained from all participants.

\section{Islet autoantibodies assay}

389 GD blood samples and 334 HT blood samples taken into the plain tubes, which were drawn at the patients' enrollment, were centrifuged at $350 \boldsymbol{g}$ and serum was stored frozen at $-80^{\circ} \mathrm{C}$ for $6.36 \pm 6.34$ months for detecting islet autoantibodies. Serum islet autoantibodies were measured by radioligand binding assay as previously described $(26,27)$, using ${ }^{35}$-labeled glutamic acid decarboxylase-65 (GAD65), protein-tyrosinephosphatase-2 (IA-2) and zinc transporter 8 (ZnT8). The plasmid containing the cDNA for cytoplasmic carboxyterminal domains (amino acid 268-369) of human ZnT8 carrying 325Arg (designated as CR) or a hybrid construct of the CR and 325Trp (CW; designated as CW-CR) with a CLFCEDPCDPSTPPGSSqGGGKDFSILLME hinge junction was generated.

Antibody levels were expressed as an immunoprecipitation index, which is defined as (sample - negative control)/(positive control - negative 


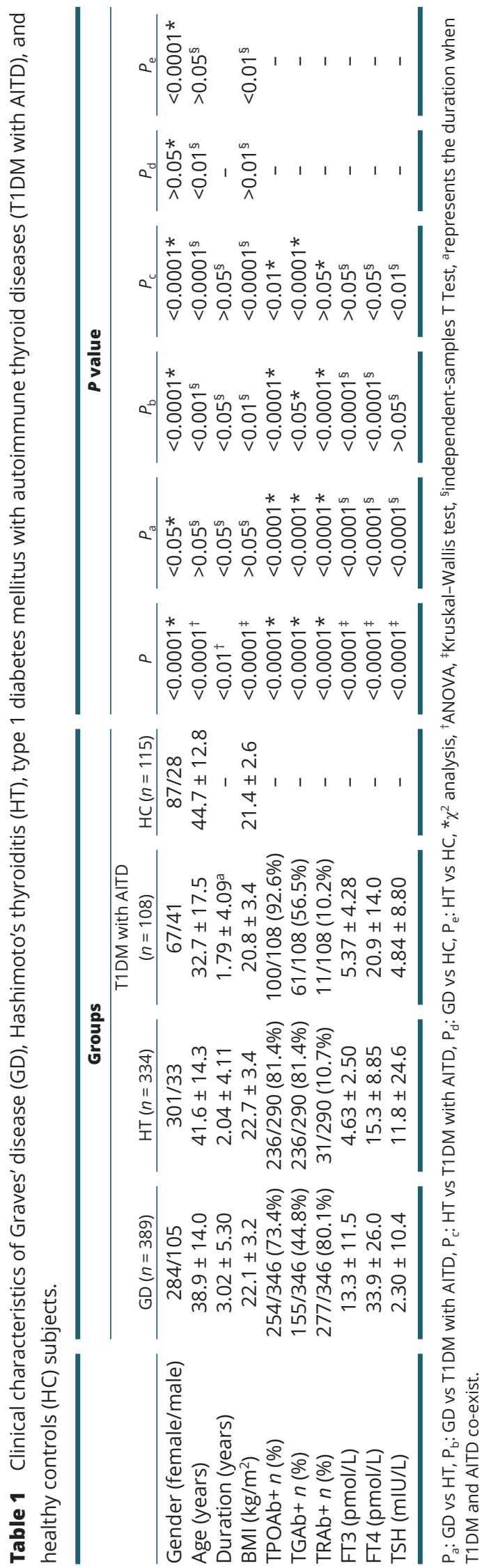

https://ec.bioscientifica.com https://doi.org/10.1530/EC-20-0650

C 2021 The authors Published by Bioscientifica Ltd control). The cut-off for positivity for GADA, IA-2A and ZnT8A was defined as a value above 0.048, 0.018, and 0.015 respectively, based on the 99th percentile of 102 ( 52 female and 50 male; aged $44.7 \pm 14.1$ years; BMI $21.4 \pm 2.6 \mathrm{~kg} / \mathrm{m}^{2}$ ), 315 (167 female and 148 male; aged $45.5 \pm 14.1$ years; BMI $\left.21.8 \pm 2.5 \mathrm{~kg} / \mathrm{m}^{2}\right)$ and 218 (110 female and 108 male; aged $46.1 \pm 14.4$ years; BMI $21.6 \pm 2.6 \mathrm{~kg} / \mathrm{m}^{2}$ ) healthy control subjects (non-diabetic individuals without known autoimmune disease and no family history of diabetes).

Our laboratory has been validated in Islet Autoantibody Standardization Program 2020 with $60.0 \%$ study sensitivity and $100 \%$ study specificity for GADA, $70.0 \%$ study sensitivity and $100 \%$ study specificity for IA-2A and $62.0 \%$ study sensitivity and $100 \%$ study specificity for ZnT8A. The coefficient of variation (CV) within-batch of measuring IA-2A, GADA, ZnT8A is 5-9, 7-9 and 4-7\%, respectively, while the CV between-batch is $8-10,5-10$ and $6-12 \%$, respectively.

\section{Thyroid function and anti-thyroid antibodies}

In this study, 346 GD and 290 HT blood samples were collected at the study entry concurrently for detecting thyroid function and anti-thyroid antibodies. Free triiodothyronine (FT3), free tetraiodothyronine (FT4), thyroid-stimulating hormone (TSH), TgAb, and TPOAb were all measured by chemiluminescence assays (Roche Diagnostics $\mathrm{GmbH}$ ), while TRAb was tested by RIA (Cisbio Bioassays, France). Reference ranges for adults are TSH, $0.270-4.20 \mathrm{mIU} / \mathrm{L}$; FT3, 3.10-6.80 $\mathrm{pmol} / \mathrm{L}$; FT4, $\quad 12.0-22.0 \quad \mathrm{pmol} / \mathrm{L} ; \quad \mathrm{TgAb}, \quad<115 \mathrm{IU} / \mathrm{mL}$; TPOAb, <34.0 IU/mL; and TRAb, 0-1.50 IU/L.

\section{Statistical analysis}

Statistical analysis was performed with SPSS 19.0 for Windows and Prism 9 for Mac. Results are shown as mean \pm S.D. or proportion (\%) or otherwise documented as positive cases or odds ratio (OR), 95\% CI. Unpaired Student's $t$-test or ANOVA was used to compare groups, and Kruska-Wallis test was used for multigroup measurement data with unequal variances. The difference between classified variables was tested using $\chi^{2}$ or Fisher's exact test if the expected number of subjects in any cell was less than five. Multiple logistic regression model was used to analyze the correlation of measurement data. Statistical significance was defined as $P<0.05$. 


\section{Results}

\section{Clinical characteristics of patients in GD, HT, T1DM with AITD and HC groups}

The clinical baselines of GD, HT, T1DM with AITD and HC groups have been described in Fig. 1A and Table 1. Among the 346 GD patients and 290 HT patients, three thyroid autoantibodies were detected currently when blood samples were collected. We observed significantly higher frequencies of either TPOAb or TGAb in the HT patients compared with the GD patients (TPOAb: $81.4 \%$ vs $73.4 \%$; TGAb: $81.4 \%$ vs $44.8 \%$, both $P<0.0001$ ), while the prevalence of TRAb was significantly higher in the GD patients compared with the HT patients $(80.1 \%$ vs $10.7 \%, P<0.0001)$. When it comes to T1DM with AITD subjects, due to the composition of diseases (98 of 108 were diagnosed as HT), there were remarkably higher frequencies of TPOAb but lower frequencies of TGAb comparing with HT patients (TPOAb: $92.6 \%$ vs $81.4 \%$, $P<0.01$; TGAb: $56.5 \%$ vs $81.4 \%, P<0.0001$, while TRAb was similarly as the HT group (10.2\% vs $10.7 \%, P>0.05)$.
The thyroid function of patients was measured when they were recruited in this study. There were great differences among FT3, FT4, TSH when comparing GD patients and HT patients, as the recruited GD patients were more prone to hyperthyroidism than HT patients (FT3: $13.3 \pm 11.5$ vs $4.63 \pm 2.50 \mathrm{pmol} / \mathrm{L}$, FT4: $33.9 \pm 26.0$ vs $15.3 \pm 8.85 \mathrm{pmol} / \mathrm{L}$, TSH: $2.30 \pm 10.4$ vs $11.8 \pm 24.6 \mathrm{mIU} / \mathrm{L}$, all $P<0.0001)$.

\section{Prevalence of islet autoantibodies in different groups}

All 389 GD' $^{\prime}$ and 334 HT patients' sera were measured for three islet autoantibodies. There were 71 (18.3\%) GD patients and 64 (19.2\%) HT patients separately positive for at least one islet autoantibody, both higher than that in the HC group $(3 / 115,2.61 \%$, both $P<0.00001)$ (Figs $1 \mathrm{~B}$ and 2D). We followed those 135 islet-autoantibodypositive AITD patients up around $8.78 \pm 1.26$ years according to their outpatient clinical records and found
A

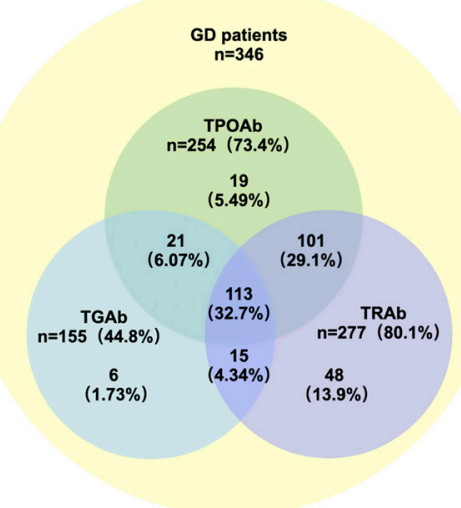

B

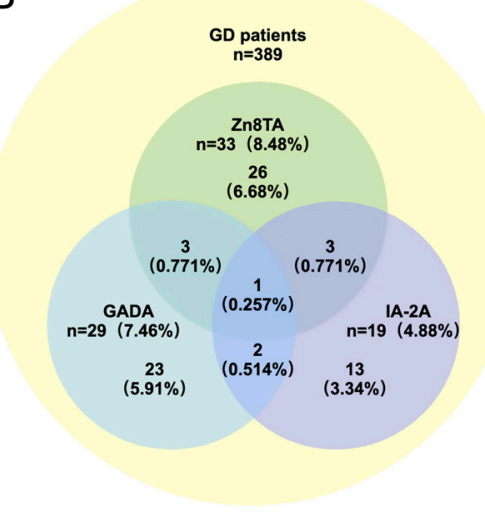

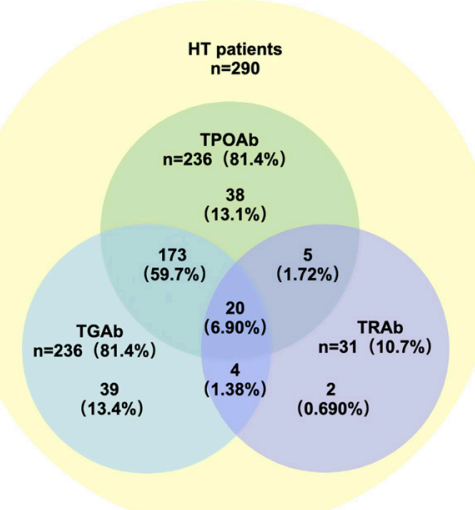

Figure 1

Frequency of islet autoantibodies and thyroid autoantibodies in Graves' disease (GD) and Hashimoto's thyroiditis (HT) patients. (A)

Frequency of thyroid autoantibodies is shown in 346 patients with GD (left) and 290 patients with HT (right). Of these, 323 (93.4\%) and 281 (96.9\%) were respectively positive for at least one thyroid autoantibody. (B) Frequency of islet autoantibodies is shown in 389 patients with GD (left) and 334 patients with HT (right). Of these, 71 $(18.3 \%)$ and 64 (19.2\%) were respectively positive for at least one islet autoantibody. https://ec.bioscientifica.com

https://doi.org/10.1530/EC-20-0650 (c) 2021 The authors Published by Bioscientifica Ltd

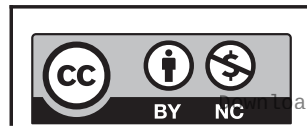

This work is licensed under a Creative Commons Attribution-NonCommercial 4.0 International License. ded from Bioscientifica.com at 04/26/2023 08:16:06AM via free access 
that three patients were diagnosed with type 2 diabetes mellitus (T2DM). One patient was diagnosed with T2DM in the same year but 2 months later followed by the diagnosis of HT, while one was diagnosed 5 years and the other one was diagnosed 9 years after HT. Interestingly, all of them were recruited in the HT group and positive for single-islet autoantibody, ZnT8A, GADA and IA-2A respectively.

The prevalence of islet autoantibodies in AITD patients (GD and HT separately) was higher than that in HC (GD vs HC: ZnT8A: $8.48 \%$ vs $1.74 \%, P<0.05$; IA-2A: $4.88 \%$ vs $0 \%, P<0.05$; GADA: $7.46 \%$ vs $0.870 \%$, $P<0.05$; 1 islet Ab: $18.3 \%$ vs $2.61 \%, P<0.0001$; HT vs HC: ZnT8A: $10.8 \%$ vs $1.74 \%, P<0.01$; IA-2A: $3.59 \%$ vs $0 \%, P<0.05$; GADA: $7.74 \%$ vs $0.870 \%, P<0.05 ; 1$ islet Ab: $19.2 \%$ vs $2.61 \%, P<0.0001)$. However, there was no difference in the positive rate between GD and HT patients (ZnT8A: $8.48 \%$ vs $10.8 \%$; IA-2A: $4.88 \%$ vs 3.59\%; GADA: $7.46 \%$ vs $7.74 \%$; $\geq 1$ islet $\mathrm{Ab}: 18.3 \%$ vs 19.2\%; all $P>0.05$ ) (Fig. 2A, B, C and D). Furthermore, the prevalence of at least two islet antibodies positive in the GD group, which in $\mathrm{HC}$ was $0 \%$, was slightly higher than the HT group without significance (Fig. 2E). In accordance with expectation, the islet autoantibodies' frequencies of patients with both T1DM and AITD were the highest, when comparing them with GD and HT subjects(ZnT8A: 42.6\%; IA-2A: 44.4\%; GADA: $74.1 \%$; $\geq 1$ islet $\mathrm{Ab}: 88.0 \%$; $\geq 2$ islet $\mathrm{Ab}: 49.1 \%$, all $P<0.0001)$.

\section{Comparison of the prevalence of islet autoantibodies in AITD subjects with high and low titer of TPOAb}

According to previous study $(28,29)$, we divided AITD patients into two subgroups according to the titer of TPOAb, namely the high-titer TPOAb (H-TPOAb) group $(\geq 100 \mathrm{IU} / \mathrm{mL}$ ) and the low-titer TPOAb (L-TPOAb ) group $(<100 \mathrm{IU} / \mathrm{mL})$. There were $217 \mathrm{H}-\mathrm{TPOAb}$ and $129 \mathrm{~L}-\mathrm{TPOAb}$ subjects in the GD group, while $197 \mathrm{H}-\mathrm{TPOAb}$ and 93 L-TPOAb subjects in the HT group respectively. Considering GD and HT as a whole AITD group, we observed an increased positive rate of IA-2A in L-TPOAb AITD patients (IA-2A: $6.76 \%$ vs $3.14 \%, P<0.05$ ). Moreover, the positive rate of GADA and at least one islet autoantibody shows no significance between L-TPOAb and H-TPOAb groups (GADA: $9.01 \%$ vs $6.28 \%, P>0.05$; $\geq 1$ islet Ab: $21.2 \%$ vs $17.9 \%, P>0.05)$. In contrast, the prevalence of $\mathrm{ZnT} 8 \mathrm{~A}$ in the H-TPOAb group was $10.9 \%(45 / 414)$, compared with $8.11 \%(18 / 222, P>0.05)$ in the L-TPOAb group (Fig. $3 \mathrm{~A})$. There was similar trend which was observed in GD and HT groups but without significance (Supplementary Fig. 1). However, the cumulative prevalence for any islet autoantibody as well as at least one islet autoantibody differed little as the titer of TPOAb increased (Fig. 3B).

In addition, according to the positivity of islet autoantibodies, we divided AITD patients into eight subgroups as presented in Supplementary Table 2. Further analyzing the level of three islet autoantibodies between
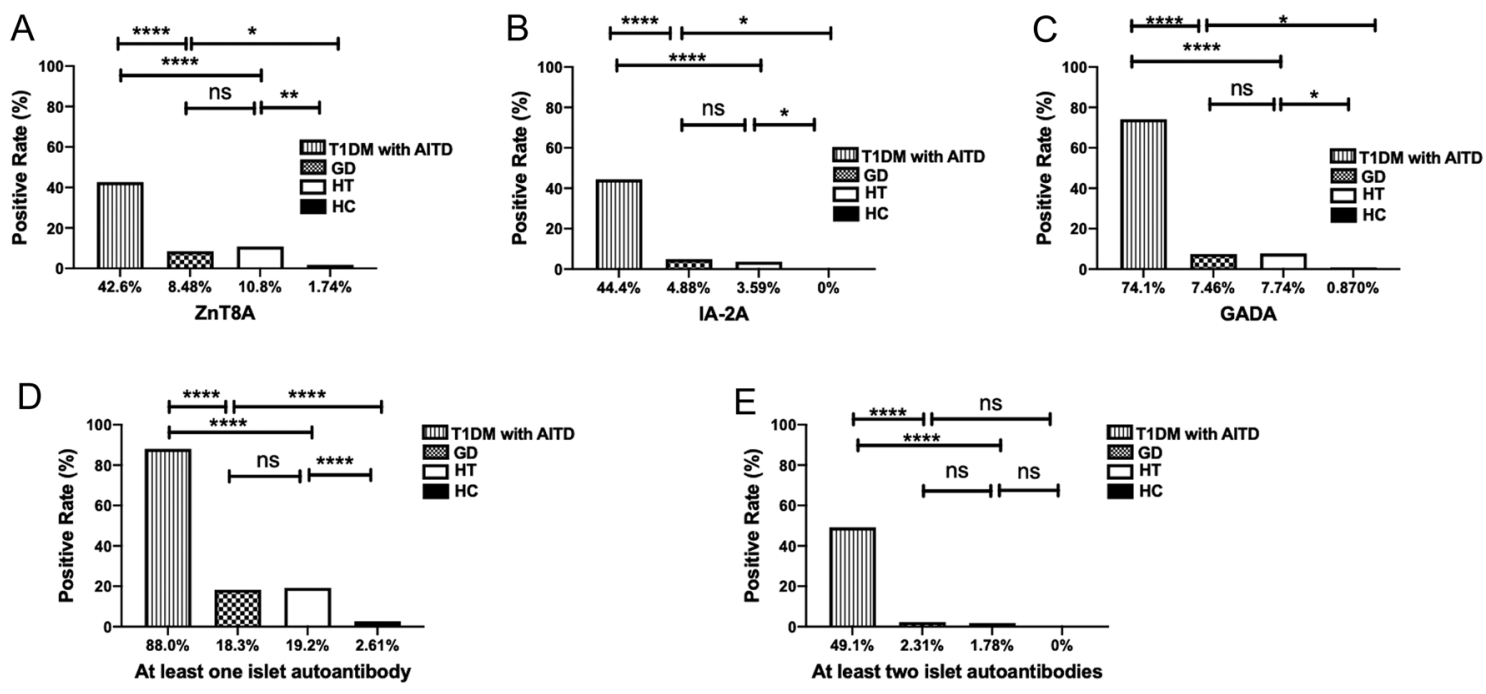

\section{Figure 2}

Frequency of islet autoantibodies in type 1 diabetes mellitus with autoimmune thyroid diseases (T1DM with AITD), Graves' disease (GD), Hashimoto's thyroiditis (HT) and healthy controls $(\mathrm{HC})$ subjects. Patients' serum was used for measuring (A) ZnT8A, (B) IA-2A, (C) GADA by radioligand binding assay. The figures show the frequency of (A) ZnT8A, (B) IA-2A, (C) GADA, (D) at least one islet autoantibody and (E) at least two islet autoantibodies in T1DM with AITD, GD, HT and HC groups. 
subgroups, a higher prevalence for TPOAb positivity between IA-2A-negative and IA2A-positive AITD patients $(72.5 \%$ vs $68.2 \%, P<0.05$; OR $=0.4037,95 \%$ CI: 0.2003-0.8300) was observed. Furthermore, a higher proportion of patients with high-titer TPOAb was found in the IA-2A-negative than that of IA-2A-positive AITD patients $(59.1 \%$ vs $35.5 \% P<0.05$; OR $=0.3806,95 \% \mathrm{CI}$ : 0.1754-0.8150). There were no statistically significant differences in the sexual proportion, subtypes of the disease, age, duration, anti-thyroid treatment, and level of either TSH or FT4 between islet autoantibody-positive and -negative AITD patients, except for the statistical decreases in the rate of BMI between IA-2A-positive and -negative AITD patients $(P<0.01, \beta=0.1506$, standard error: $0.05790, \operatorname{EXP}(\beta)=1.163,95 \% \mathrm{CI}=1.039-1.31$ ). These findings suggested that the increasing level of islet autoimmunity was independent of subtypes of the disease, gender, age, duration, anti-thyroidal treatment, TGAb positivity and thyroid function, but the BMI as well as the positivity and titer of TPOAb were related with IA-2A.

\section{Discussion}

The most common coexisting organ-specific autoimmune disease in patients with T1DM is AITD. In our study, based on a larger cohort of 723 Chinese AITD patients, the prevalence of ZnT8A was as high as 8.48 and $10.8 \%$ in GD and HT patients, respectively, which was significantly higher than that in HC and lower than T1DM with AITD. It was also a novel finding that the prevalence rate of ZnT8A was much higher than that of GADA (GD:7.46\%, HT: $7.74 \%$ ) and IA-2A (GD:4.88\%, HT: 3.59\%) in AITD patients, as GADA in the previous studies was the highest diabetes-associated antibody (17, 18, 19, 26, 30). Even though there were only three patients positive for singleislet antibody developing T2DM during $8.78 \pm 1.26$ followed-up years, the diabetes progression may still prove the relations between islet autoimmunity and diabetes in our study. We diagnosed them based on the clinical history and treatment regimen. Despite the fact that all of three patients were insulin-independent since diagnosed and without an onset by diabetic ketoacidosis (DKA) which supports the diagnosis of T2DM, we still could not exclude the possibility of latent autoimmune diabetes in adults (LADA). Genetic analysis and dynamic monitoring of fasting C-peptide will be carried on in our further long-term study.

ZnT8A is the recently identified new marker of autoimmunity in diabetes. Jonsdottir et al. (22) recruited
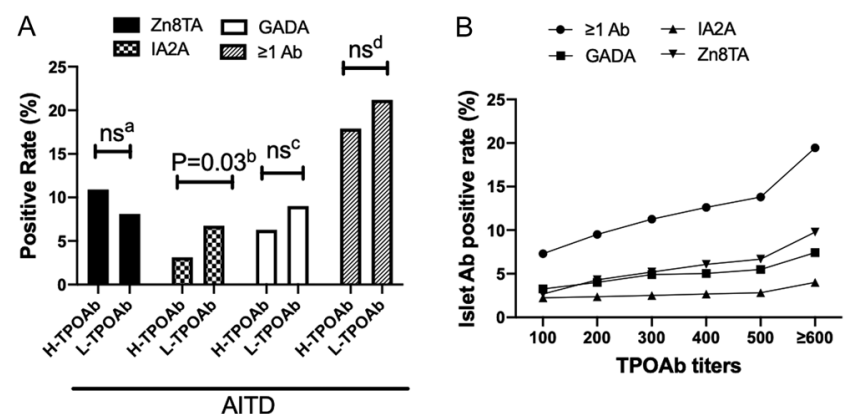

\section{Figure 3}

Frequency of islet autoantibodies in autoimmune thyroid diseases (AITD) (including Graves' disease, Hashimoto's thyroiditis) subjects with high titer TPOAb and low titer TPOAb. According to TPOAb $\geq 100 \mathrm{IU} / \mathrm{mL}$ and $<100$ $\mathrm{IU} / \mathrm{mL}$, we divided the AITD (containing GD and HT) into high titer TPOAb (H-TPOAb) and low titer TPOAb (L-TPOAb) groups separately. (A) shows the positive rate of ZnT8A (H-TPOAb vs L-TPOAb: $10.9 \%$ vs $8.11 \%$ ), IA-2A (3.14\% vs $6.76 \%)$, GADA(6.28\% vs $9.01 \%)$ and at least one islet autoantibody (17.9\% vs $21.2 \%$ ) in H-TPOAb and L-TPOAb groups. (B) shows the cumulative positive rates of ZnT8A, IA-2A, GADA and at least one islet autoantibody in AITD subjects with different TPOAb titers. ${ }^{a} P=0.33$, odds ratio $=1.382,95 \% \mathrm{Cl}=0.7976-2.480 .{ }^{\mathrm{b}} P=0.03$, odds ratio $=0.4474$, $95 \% \mathrm{Cl}=0.2170-0.9709$. ${ }^{c} P=0.20$, odds ratio $=0.6768,95 \% \mathrm{Cl}=$ $0.3645-1.252 .{ }^{d} P=0.34$, odds ratio $=0.8104,95 \% \mathrm{Cl}=0.5412-1.216$.

278 patients with newly diagnosed GD for detecting GADA, IA-2A, three variants of ZnT8A, and they observed that $7.6 \%$ of them were positive for ZnT8A at diagnosis. Rydzewska's study, which consisted of 44 children with GD, 65 children with HT, 199 children with T1DM with or without AITDs and 58 control children (23), reported the overall prevalence of ZnT8A with AITD was 9.2\%, with a similar prevalence of ZnT8A in both GD (9.1\%) and HT (9.2\%). Previous evidence has already shown that ZnT8A is widely distributed in a lot of endocrine or autoimmune disorders. As in the report based on 223 new-onset T1DM patients (24), ZnT8A occurred in $60-80 \%$ of them, while 12 of 39 (30.9\%) non-diabetic patients developed T1DMrelated celiac diseases. In accord with these findings, our study illustrated that the prevalence of ZnT8A in nondiabetic GD or HT patients was significantly higher than that in HC but lower than T1DM with AITD patients as expected, which pointed out the perspective on ZnT8A's representativeness in endocrine autoimmune diseases. Rydzewska et al. (23) found that the strong ZnT8 expression could be observed in almost all of the thyroid follicular cells and some of the cytoplasm and the perinuclear area of the hyperplastic C cells. In keeping with that, ZnT8 was identified as a major autoantigen in T1DM and was localized in insulin secretory granules $(24,31)$, which was expressed in other secretory cell types of murine like pancreatic $\alpha$ islet cells, thyroid cubical cells and adrenal gland cortex cells as well (32). Our data further confirmed

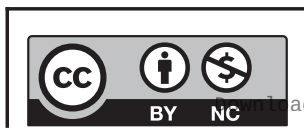

This work is licensed under a Creative Commons Attribution-NonCommercial 4.0 International License. ded from Bioscientifica.com at 04/26/2023 08:16:06AM 
the widespread role of ZnT8 in the endocrine system mentioned above $(22,24,32)$.

GADA and IA-2A are recommended markers for the suspected diagnosis of T1DM or for the prediction of T1DM in research settings (33). Consistent with previous studies $(17,19,20,34,35)$, our study demonstrated that the prevalence of GADA and IA-2A was ranging from 7-8 and 3-5\%, respectively in AITD patients. A recent Chinese study, in which 190 T1DM and 135 LADA patients participated, revealed that a high level of GADA could be a strong predictor of the development of thyroid autoimmunity in patients with autoimmune diabetes (36) as well as the finding of Lethagen et al. (37) demonstrated that GADA positivity could be a marker of subclinical insulitis in a study on 441 non-diabetic patients with autoimmune thyroiditis. Both of the studies indicated the potential of GADA in predicting concurrent thyroid autoimmunity and islet autoimmunity. Additionally, Pilia's study (19) illustrated that IA-2A was significantly prevalent in 236 non-obese Sardinian children with autoimmune thyroiditis, which is consistent with our results to some degree, however, their prevalence of GADA showed no significant differences compared with HC. The composition of patients and differences of race may explain the discordance with our results.

According to the titer of TPOAb, we compared the prevalence of ZnT8A, GADA and IA-2A in H-TPOAb and L-TPOAb groups. Only a higher positive rate of IA-2A $(P<0.05, \mathrm{OR}=0.4474,95 \% \mathrm{CI}=0.2170-0.9709)$ was observed in L-TPOAb patients. Similarly, the positive rate of GADA seemed higher in the L-TPOAb group $(P>0.05$, $\mathrm{OR}=0.6768,95 \% \mathrm{CI}=0.3645-1.252)$. In contrast, ZnT8A positivity was higher in the H-TPOAb group but without significance $(P>0.05, \mathrm{OR}=1.382,95 \% \mathrm{CI}=0.7976-2.480)$. Therefore, we infer that AITD patients with H-TPOAb are more likely to develop ZnT8A positivity, meanwhile, patients with L-TPOAb develop GADA and IA-2A. Moreover, despite the positive rate of ZnT8A, GADA or IA-2A did not vary much with TPOAb titer increasing, we still could observe the increasing trend along with the TPOAb titer rising. Rogowicz-Frontczak et al. (25) revealed an association among positive ZnT8A, GADA and positive titer of TPOAb in his study which consisted of 80 nonobese adults diagnosed as LADA, and Jonsdottir et al. (11) also demonstrated an association between two thyroid autoantibodies (TPOAb and TGAb) and ZnT8A or GADA positivity in a cohort of 2433 newly diagnosed T1DM children. Unfortunately, no statistical differences were exhibited in the prevalence of ZnT8A and GADA comparing high and low-TPOAb titer in our study; longitudinal studies based on a larger follow-up population are necessary for the future to determine the predicted value of both GADA and ZnT8A. Interestingly, the significant differences of the TPOAb-positive proportion as well as the H-TPOAb proportion between IA-2A-negative and -positive patients (both $P<0.05$ ) further indicate the relations between IA-2A and TPOAb titers.

Our study is powered by a large number of GD, HT, T1DM with AITD and HC patients of whom thyroid autoantibodies and islet autoantibodies were detected. However, we still acknowledge the limitations. First of all, our previous study (26) of 600 Chinese T1DM patients shows that the positive rates of ZnT8A in patients who carried DRB1*0901, IA2A in patients who carried DRB1*0405 and A*1101-DRB1*0901 and GADA in patients who carried DRB1*0901 and A* 2402-DRB1* 0901 were significantly higher than those that not carried. HLA genotyping and association between HLA haplotype and islet autoantibodies should be continued in our future research. Secondly, the lack of dynamic monitoring of patients' C-peptide and blood glucose metabolic index limited the observation on the changes of islet $\beta$-cell function and anti-islet autoimmune status. Thirdly, the discordance of sexual and age proportion among patients in different groups may decrease the statistical power, thus, we should further expand the subjects' population to avoid it in the future long-term cohort. Last but not least, some recent studies have shown that patients with AITD are at increased risk of developing T1DM $(19,38)$, however, the presence of any one single autoantibody appeared not to be predictive for the disease $(39,40,41)$. Unfortunately, there were only three out of 135 patients who were positive for single-islet autoantibody-developed diabetes and clinically diagnosed as T2DM. Research on investigating the potential relations between the islet autoantibody and special types of diabetes (such as LADA) should be launched in the future.

\section{Conclusions}

In conclusion, an increased prevalence of ZnT8A, as well as a high prevalence of islet autoimmunity, was found in both GD and HT patients. It indicates that there is a potential link between thyroid autoimmunity and islet autoimmunity. We suggest, therefore, that patients with AITD may need screening for islet autoantibodies, and those who are positive for at least one islet autoantibodies need long-term follow-up for islet function and blood glucose.

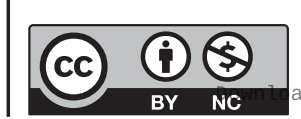

This work is licensed under a Creative Commons Attribution-NonCommercial 4.0 International License. ded from Bioscientifica.com at 04/26/2023 08:16:06AM 


\section{Supplementary materials}

This is linked to the online version of the paper at https://doi.org/10.1530/ EC-20-0650.

\section{Declaration of interest}

The authors declare that there is no conflict of interest that could be perceived as prejudicing the impartiality of the research reported.

\section{Funding}

This work is supported by the grant from the National Natural Science Foundation of China (No. 81270897), the Jiangsu Provincial Special Program of Medical Science (BL2012026) and the Project Funded by the Priority Academic Program Development of Jiangsu Higher Education Institutions.

\section{Author contribution statement}

$\mathrm{CY}$ and $\mathrm{YJN}$ designed and wrote the manuscript and performed most experiments. YT and ZXQ contributed to design of the study and reviewed and edited the manuscript. YLP help to take control of the autoantibodies assay. All authors reviewed and take full responsibility for the contents of the manuscript. YT is the guarantor of this work and, as such, had full access to all the data in the study and takes responsibility for the integrity of the data and the accuracy of the data analysis.

\section{Acknowledgements}

The authors express their sincere appreciation to George Eisenbarth, John Hutton and Howard Davidson (Barbara Davis Center of Childhood Diabetes, University of Colorado, CO, USA) for their tutoring and guidance.

\section{References}

1 Weetman AP. Graves' disease. New England Journal of Medicine 2000343 1236-1248. .(https://doi.org/10.1056/ NEJM200010263431707)

2 Pearce EN, Farwell AP \& Braverman LE. Thyroiditis. New England Journal of Medicine 2003348 2646-2655. . (https://doi.org/10.1056/ NEJMra021194)

3 Golden B, Levin L, Ban Y, Concepcion E, Greenberg DA \& Tomer Y. Genetic analysis of families with autoimmune diabetes and thyroiditis: evidence for common and unique genes. Journal of Clinical Endocrinology and Metabolism 200590 4904-4911. .(https:// doi.org/10.1210/jc.2004-2236)

4 Barker JM, Yu J, Yu L, Wang J, Miao D, Bao F, Hoffenberg E, Nelson JC, Gottlieb PA, Rewers M, et al. Autoantibody 'subspecificity' in type 1 diabetes: risk for organ-specific autoimmunity clusters in distinct groups. Diabetes Care 200528 850-855. .(https://doi. org/10.2337/diacare.28.4.850)

5 De Block CE, De Leeuw IH, Vertommen JJ, Rooman RP, Du Caju MV, Van Campenhout CM, Weyler JJ, Winnock F, Van Autreve J, Gorus FK, et al. Beta-cell, thyroid, gastric, adrenal and coeliac autoimmunity and HLA-DQ types in type 1 diabetes. Clinical and Experimental Immunology 2001126 236-241. (https://doi. org/10.1046/j.1365-2249.2001.01668.x)

6 Eisenbarth GS \& Gottlieb PA. Autoimmune polyendocrine syndromes. New England Journal of Medicine $20043502068-2079$. .(https://doi.org/10.1056/NEJMra030158)

7 Jenkins RC \& Weetman AP. Disease associations with autoimmune thyroid disease. Thyroid 200212 977-988. . (https://doi. org/10.1089/105072502320908312)
8 Eisenbarth GS. Type I diabetes mellitus: a chronic autoimmune disease. New England Journal of Medicine 1986314 1360-1368. .(https://doi.org/10.1056/NEJM198605223142106)

9 Brorsson CA \& Pociot F. Shared genetic basis for type 1 diabetes, islet autoantibodies, and autoantibodies associated with other immunemediated diseases in families with type 1 diabetes. Diabetes Care 2015 38 (Supplement 2) S8-S13. .(https://doi.org/10.2337/dcs15-2003)

10 Holl RW, Bohm B, Loos U, Grabert M, Heinze E \& Homoki J. Thyroid autoimmunity in children and adolescents with type 1 diabetes mellitus. Effect of age, gender and HLA type. Hormone Research 1999 52 113-118. .(https://doi.org/10.1159/000023446)

11 Jonsdottir B, Andersson C, Carlsson A, Delli A, Forsander G, Ludvigsson J, Marcus C, Samuelsson U, Ortqvist E, Lernmark A, et al. Thyroid autoimmunity in relation to islet autoantibodies and HLA-DQ genotype in newly diagnosed type 1 diabetes in children and adolescents. Diabetologia 201356 1735-1742. (https://doi. org/10.1007/s00125-013-2934-9)

12 Hanukoglu A, Mizrachi A, Dalal I, Admoni O, Rakover Y, Bistritzer Z, Levine A, Somekh E, Lehmann D, Tuval M, et al. Extrapancreatic autoimmune manifestations in type 1 diabetes patients and their first-degree relatives: a multicenter study. Diabetes Care 200326 1235-1240. (https://doi.org/10.2337/diacare.26.4.1235)

13 Jaeger C, Hatziagelaki E, Petzoldt R \& Bretzel RG. Comparative analysis of organ-specific autoantibodies and celiac disease associated antibodies in type 1 diabetic patients, their first-degree relatives, and healthy control subjects. Diabetes Care 200124 27-32. (https://doi.org/10.2337/diacare.24.1.27)

14 Bonifacio E, Mayr A, Knopff A \& Ziegler AG. Endocrine autoimmunity in families with type 1 diabetes: frequent appearance of thyroid autoimmunity during late childhood and adolescence. Diabetologia 200952 185-192. (https://doi.org/10.1007/s00125-0081206-6)

$15 \mathrm{Ng}$ WY, Thai AC, Lui KF, Yeo PP \& Cheah JS. IgG-class insulin autoantibodies in autoimmune thyroid disease. International Archives of Allergy and Applied Immunology 199091 431-436. (https://doi. org/10.1159/000235154)

16 Yamaguchi Y, Chikuba N, Ueda Y, Yamamoto H, Yamasaki H, Nakanishi T, Akazawa S \& Nagataki S. Islet cell antibodies in patients with autoimmune thyroid disease. Diabetes 199140 319-322. (https://doi.org/10.2337/diab.40.3.319)

17 Kawasaki E, Abiru N, Yano M, Uotani S, Matsumoto K, Matsuo H, Yamasaki H, Yamamoto H, Yamaguchi Y \& Akazawa S. Autoantibodies to glutamic acid decarboxylase in patients with autoimmune thyroid disease: relation to competitive insulin autoantibodies. Journal of Autoimmunity 19958 633-643. (https://doi. org/10.1006/jaut.1995.0047)

18 Maugendre D, Vérité F, Guilhem I, Genetet B, Allannic H \& Delamaire M. Anti-pancreatic autoimmunity and Graves' disease: study of a cohort of 600 Caucasian patients. European Journal of Endocrinology 1997137 503-510. (https://doi.org/10.1530/ eje.0.1370503)

19 Pilia S, Casini MR, Cambuli VM, Ibba A, Civolani P, Zavattari P, Incani M, Mossa P, Baroni MG, Mariotti S, et al. Prevalence of Type 1 diabetes autoantibodies (GAD and IA2) in Sardinian children and adolescents with autoimmune thyroiditis. Diabetic Medicine 201128 896-899. (https://doi.org/10.1111/j.1464-5491.2011.03313.x)

20 Moriguchi M, Noso S, Kawabata Y, Yamauchi T, Harada T, Komaki K, Babaya N, Hiromine Y, Ito H, Yamagata S, et al. Clinical and genetic characteristics of patients with autoimmune thyroid disease with anti-islet autoimmunity. Metabolism: Clinical and Experimental 2011 60 761-766. (https://doi.org/10.1016/j.metabol.2010.07.025)

21 Sallorenzo C, Silva R, Kasamatsu T \& Dib S. Prevalence of pancreatic autoantibodies in non-diabetic patients with autoimmune thyroid disease and its relation to insulin secretion and glucose tolerance. Archives of Endocrinology and Metabolism 201761 361-366. (https:// doi.org/10.1590/2359-3997000000280)

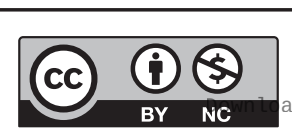

This work is licensed under a Creative Commons Attribution-NonCommercial 4.0 International License. ded from Bioscientifica.com at 04/26/2023 08:16:06AM 
22 Jonsdottir B, Jönsson I \& Lantz M. Prevalence of diabetes and presence of autoantibodies against zinc transporter 8 and glutamic decarboxylase at diagnosis and at follow up of Graves' disease. Endocrine 201964 48-54. (https://doi.org/10.1007/s12020-019. 01852-w)

23 Rydzewska M, Michalak J, Bossowska A, Chen S, Black S, Powell M, Furmaniak J, Rees Smith B \& Bossowski A. Analysis of diabetesassociated autoantibodies in children and adolescents with autoimmune thyroid diseases. Journal of Pediatric Endocrinology and Metabolism 201932 355-361. (https://doi.org/10.1515/jpem-20180367)

24 Wenzlau JM, Juhl K, Yu L, Moua O, Sarkar SA, Gottlieb P, Rewers M, Eisenbarth GS, Jensen J, Davidson HW, et al. The cation efflux transporter ZnT8 (Slc30A8) is a major autoantigen in human type 1 diabetes. PNAS 2007104 17040-17045. (https://doi.org/10.1073/ pnas.0705894104)

25 Rogowicz-Frontczak A, Zozuliłska-Ziołkiewicz D, Litwinowicz M, Niedźwiecki P, Wyka K \& Wierusz-Wysocka B. Are zinc transporter type 8 antibodies a marker of autoimmune thyroiditis in non-obese adults with new-onset diabetes? European Journal of Endocrinology 2014170 651-658. (https://doi.org/10.1530/EJE-13-0901)

$26 \mathrm{Gu}$ Y, Zhang M, Chen H, Wang Z, Xing C, Yang H, Xu X, Liu Y, Zhou Z, Yu L, et al. Discordant association of islet autoantibodies with high-risk HLA genes in Chinese type 1 diabetes. Diabetes/ Metabolism Research and Reviews 201127 899-905. (https://doi. org/10.1002/dmrr.1270)

27 Liu J, Bian L, Ji L, Chen Y, Chen H, Gu Y, Ma B, Gu W, Xu X, Shi Y, et al. The heterogeneity of islet autoantibodies and the progression of islet failure in type 1 diabetic patients. Science China: Life Sciences 201659 930-939. (https://doi.org/10.1007/s11427-016-5052-3)

28 Paparodis RD, Bantouna D, Karvounis E, Imam S \& Jaume JC. Higher TSH is not associated with thyroid cancer risk in the presence of thyroid autoimmunity. Journal of Clinical Endocrinology and Metabolism 2020105 e2389-e2397. (https://doi.org/10.1210/clinem/dgaa237)

29 Imam S, Dar P, Paparodis R, Almotah K, Al-Khudhair A, Hasan SAM, Salim N \& Jaume JC. Nature of coexisting thyroid autoimmune disease determines success or failure of tumor immunity in thyroid cancer. Journal for ImmunoTherapy of Cancer 2019 7 3. (https://doi. org/10.1186/s40425-018-0483-y)

30 Pietropaolo M, Peakman M, Pietropaolo SL, Zanone MM, Foley TP, Becker DJ \& Trucco M. Combined analysis of GAD65 and ICA512(IA-2) autoantibodies in organ and non-organ-specific autoimmune diseases confers high specificity for insulin-dependent diabetes mellitus. Journal of Autoimmunity 199811 1-10. (https://doi org/10.1006/jaut.1997.0170)

31 Chimienti F, Devergnas S, Favier A \& Seve M. Identification and cloning of a beta-cell-specific zinc transporter, ZnT-8, localized into insulin secretory granules. Diabetes 200453 2330-2337. (https://doi. org/10.2337/diabetes.53.9.2330)

32 Murgia C, Devirgiliis C, Mancini E, Donadel G, Zalewski P \& Perozzi G. Diabetes-linked zinc transporter ZnT8 is a homodimeric protein expressed by distinct rodent endocrine cell types in the pancreas and other glands. Nutrition, Metabolism, and Cardiovascular Diseases 200919 431-439. (https://doi.org/10.1016/j. numecd.2008.09.004)

33 Winter WE \& Schatz DA. Autoimmune markers in diabetes. Clinical Chemistry 201157 168-175. (https://doi.org/10.1373/ clinchem.2010.148205)

34 Primo ME, Niepomniszcze H, Poskus E, Sala MS, Guaita SS, Sica MP, Villalba A, Cardoso A \& Bruno OD. Frequency of pancreatic beta-cell autoimmunity markers in patients with autoimmune thyroid disease. Medicina 200868 37-42.

35 Jonsdottir B, Larsson C, Carlsson A, Forsander G, Ivarsson SA, Lernmark A, Ludvigsson J, Marcus C, Samuelsson U, Örtqvist E, et al. Thyroid and islet autoantibodies predict autoimmune thyroid disease at Type 1 diabetes diagnosis. Journal of Clinical Endocrinology and Metabolism 2017102 1277-1285. (https://doi.org/10.1210/jc.20162335)

36 Jin P, Huang G, Lin J, Yang L, Xiang B, Zhou W \& Zhou Z. High titre of antiglutamic acid decarboxylase autoantibody is a strong predictor of the development of thyroid autoimmunity in patients with type 1 diabetes and latent autoimmune diabetes in adults. Clinical Endocrinology 201174 587-592. (https://doi.org/10.1111/j.13652265.2011.03976.x)

37 Lethagen AL, Ericsson UB, Hallengren B, Groop L \& Tuomi T. Glutamic acid decarboxylase antibody positivity is associated with an impaired insulin response to glucose and arginine in nondiabetic patients with autoimmune thyroiditis. Journal of Clinical Endocrinology and Metabolism 200287 1177-1183. (https://doi. org/10.1210/jcem.87.3.8343)

38 Taniyama M, Kasuga A, Nagayama C \& Ito K. Occurrence of type 1 diabetes in Graves' disease patients who are positive for antiglutamic acid decarboxylase antibodies: an 8-year followup study. Journal of Thyroid Research 20102011306487 . (https://doi. org/10.4061/2011/306487)

39 Gorus FK, Goubert P, Semakula C, Vandewalle CL, De Schepper J, Scheen A, Christie MR \& Pipeleers DG. IA-2-autoantibodies complement GAD65-autoantibodies in new-onset IDDM patients and help predict impending diabetes in their siblings. The Belgian Diabetes Registry. Diabetologia 199740 95-99. (https://doi. org/10.1007/s001250050648)

40 Bingley PJ, Bonifacio E, Williams AJ, Genovese S, Bottazzo GF \& Gale EA. Prediction of IDDM in the general population: strategies based on combinations of autoantibody markers. Diabetes 199746 1701-1710. (https://doi.org/10.2337/diab.46.11.1701)

41 Schlosser M, Strebelow M, Wassmuth R, Arnold ML, Breunig I, Rjasanowski I, Ziegler B \& Ziegler M. The Karlsburg type 1 diabetes risk study of a normal schoolchild population: association of betacell autoantibodies and human leukocyte antigen-DQB1 alleles in antibody-positive individuals. Journal of Clinical Endocrinology and Metabolism 200287 2254-2261. (https://doi.org/10.1210/ jcem.87.5.8491)

Received in final form 24 February 2021

Accepted 28 April 2021

Accepted Manuscript published online 28 April 2021 https://ec.bioscientifica.com https://doi.org/10.1530/EC-20-0650 (c) 2021 The authors Published by Bioscientifica Ltd

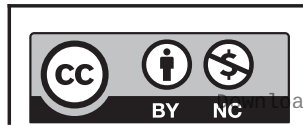

This work is licensed under a Creative Commons Attribution-NonCommercial 4.0 International License. ded from Bioscientifica.com at 04/26/2023 08:16:06AM 